Pacific

Journal of

Mathematics

ON KUIPER'S QUESTION WHETHER TAUT SUBMANIFOLDS ARE ALGEBRAIC

Thomas E. Cecil, Quo-Shin Chi And Gary R. Jensen

Volume $234 \quad$ No. 2

February 2008 


\title{
ON KUIPER'S QUESTION WHETHER TAUT SUBMANIFOLDS ARE ALGEBRAIC
}

\author{
Thomas E. Cecil, Quo-Shin Chi And GARY R. Jensen
}

\begin{abstract}
We prove that any connected proper Dupin hypersurface in $\mathbb{R}^{n}$ is analytic algebraic and is an open subset of a connected component of an irreducible algebraic set. From this we also prove that every taut submanifold of dimension $m \leq 4$ is algebraic by exploring a finiteness condition.
\end{abstract}

\section{Introduction}

An embedding $f$ of a compact, connected manifold $M$ into Euclidean space $\mathbb{R}^{n}$ is taut if every nondegenerate (Morse) Euclidean distance function $L_{p}: M \rightarrow \mathbb{R}$ such that $L_{p}(x)=d(f(x), p)^{2}$ for $p \in \mathbb{R}^{n}$ has $\beta\left(M, \mathbb{Z}_{2}\right)$ critical points on $M$, where $\beta\left(M, \mathbb{Z}_{2}\right)$ is the sum of the $\mathbb{Z}_{2}$-Betti numbers of $M$. That is, $L_{p}$ is a perfect Morse function on $M$.

We can also consider taut embeddings into $S^{n}$, in which case we use spherical distance functions instead of Euclidean distance functions. Tautness is preserved by stereographic projection, and so the theories in the two ambient spaces are essentially the same.

A connected hypersurface $M$ in $\mathbb{R}^{n}$ is said to be Dupin if along each curvature surface of $M$, the corresponding principal curvature is constant. $M$ is called proper Dupin if, in addition, the number $g$ of distinct principal curvatures is constant on $M$. Thorbergsson [1983] proved every compact proper Dupin hypersurface is taut.

Many taut submanifolds, for example, the cyclides of Dupin [Banchoff 1970], Veronese manifolds [Cecil and Ryan 1985], and isoparametric submanifolds [Terng 1985], are known to be algebraic; Kuiper [1984] asked whether all taut submanifolds of $\mathbb{R}^{n}$ must be real algebraic. In the 1980's, the affirmative answer to this question was widely thought to be true, but a proof was never published. In particular, Ulrich Pinkall [1984] sent the first author a letter containing a sketch of a proof that proper Dupin hypersurfaces are algebraic, and we thank Professor Pinkall for allowing us to use his approach to the problem in our work here.

MSC2000: 53C40, 53C42.

Keywords: Dupin hypersurface, taut submanifold, semialgebraic set.

Cecil was partially supported by NSF Grant No. DMS-0405529. Chi was partially supported by NSF Grant No. DMS-0103838. Jensen was partially supported by NSF Grant No. DMS-0604236. 
Pinkall's sketch led us to the local parametrization (3-3) of a general proper Dupin hypersurface. We then used ideas from real algebraic geometry to show that a connected proper Dupin hypersurface is contained in a connected component of an irreducible algebraic subset of $\mathbb{R}^{n}$. There are still issues to be resolved to settle this question of Kuiper's, however, because arbitrary taut submanifolds are Dupin, but not necessarily proper Dupin; see [Pinkall 1986].

In this paper, we prove the affirmative answer to Kuiper's question for manifolds of dimension $m \leq 4$. (The result in dimensions $m \leq 2$ is due to Banchoff [1970].)

\section{Algebraic preliminaries}

A semialgebraic subset of $\mathbb{R}^{n}$ is one which is a finite union of sets of the form $\bigcap_{j}\left\{x \in \mathbb{R}^{n}: F_{j}(x) * 0\right\}$, where $*$ is either $<$ or $=, F_{j}$ is a polynomial over $\mathbb{R}^{n}$, and the intersection is finite.

It follows from the definition that the complement of a semialgebraic set is semialgebraic, and hence a semialgebraic set take away another semialgebraic set leaves a semialgebraic set. Moreover, the projection $\pi: \mathbb{R}^{n} \rightarrow \mathbb{R}^{k}$ sending $x \in \mathbb{R}^{n}$ to its first $k$ coordinates maps a semialgebraic set to a semialgebraic set. As a corollary, the (topological) closure and interior of a semialgebraic set are semialgebraic.

A map $f: S \subset \mathbb{R}^{n} \rightarrow \mathbb{R}^{k}$ is semialgebraic if its graph in $\mathbb{R}^{n} \times \mathbb{R}^{k}$ is a semialgebraic set. As a result, the image of a semialgebraic map $f: S \subset \mathbb{R}^{n} \rightarrow \mathbb{R}^{k}$ is semialgebraic via the composition graph $(f) \subset \mathbb{R}^{n} \times \mathbb{R}^{k} \rightarrow \mathbb{R}^{k}$, where the last map is the projection onto the second summand.

A Nash function is a $C^{\infty}$ semialgebraic map from an open semialgebraic subset of $\mathbb{R}^{n}$ to $\mathbb{R}$. A real analytic function $f$ defined on an open semialgebraic subset $U$ of $\mathbb{R}^{n}$ is analytic algebraic if it solves a polynomial equation on $U$ of the form

$$
a_{0}(x) f^{s}(x)+a_{1}(x) f^{s-1}(x)+\cdots+a_{s}(x)=0,
$$

where $a_{0}(x) \neq 0, a_{1}(x), \ldots, a_{s}(x)$ are polynomials over $\mathbb{R}^{n}$. A significant result in the subject is that these two concepts are equivalent; see [Bochnak et al. 1998, p. 165]. A slight generalization of the single-variable case [p. 54] shows that the partial derivatives of any Nash function are again Nash functions.

Let $S$ be a semialgebraic subset of $\mathbb{R}^{n}$. The dimension of $S$, denoted $\operatorname{dim} S$, is the dimension of the ring $R=\mathbb{R}\left[x^{1}, \cdots, x^{n}\right] / \mathscr{I}(S)$, the quotient ring of the polynomial ring modulo the ideal generated by $S$. It is the maximal length of chains of prime ideals of $R$. As usual, when $S$ is a semialgebraic subset of $\mathbb{R}^{n}$ that is a $C^{\infty}$ submanifold of $\mathbb{R}^{n}$ of dimension $d$, then $\operatorname{dim} S=d$; see again [p. 54].

We denote the topological closure of $S$ by $\bar{S}^{\text {top }}$. Denote the Zariski closure of $S$ by $\bar{S}^{\text {zar }}$. It is the smallest algebraic set containing $S$. The important property that 
plays a central role in our arguments to follow is that if $S$ is a semialgebraic subset of $\mathbb{R}^{n}$, then $\operatorname{dim} S=\operatorname{dim}\left(\bar{S}^{\text {top }}\right)=\operatorname{dim}\left(\bar{S}^{\text {zar }}\right)$; see [Bochnak et al. 1998, p. 50].

Lemma 1. Let $M \subset \mathbb{R}^{n}$ be a connected analytic submanifold and let $U \subset M$ be a connected open subset of $M$. If $U$ is a semialgebraic subset of $\mathbb{R}^{n}$, then $M$ is an open subset of a connected component of the irreducible algebraic set $\bar{U}^{z a r}$.

The proof uses the fact that the Zariski closure $\bar{U}^{\mathrm{zar}}$ of $U$ is irreducible, because $\mathscr{I}\left(\bar{U}^{\text {zar }}\right)=\mathscr{I}(U)$ is a prime ideal. Hence $M \subset \mathscr{L}(\mathscr{I}(U))=\bar{U}^{\text {zar }}$, where $\mathscr{L}$ denotes the zero set of an ideal.

A semialgebraic subset $M$ of $\mathbb{R}^{m}$ is a Nash submanifold of $\mathbb{R}^{m}$ of dimension $n$ if for every point $p$ of $M$, there exists a Nash diffeomorphism $\psi$ from an open semialgebraic neighborhood $U$ of the origin in $\mathbb{R}^{m}$ into an open semialgebraic neighborhood $V$ of $p$ in $\mathbb{R}^{m}$ such that $\psi(0)=p$ and $\psi\left(\left(\mathbb{R}^{n} \times\{0\}\right) \cap U\right)=M \cap V$. Here, by a Nash diffeomorphism $\psi$ we mean that the coordinate functions of $\psi$ and $\psi^{-1}$ are Nash functions.

Let $M$ be a Nash submanifold of $\mathbb{R}^{m}$. A mapping $f: M \rightarrow \mathbb{R}$ is a Nash mapping if it is semialgebraic, and for every $\psi$ in the preceding definition, $f \circ \psi$ restricted to $\mathbb{R}^{n} \cap U$ is a Nash function. As usual, if $d f$ is never zero at all points of $f^{-1}(p)$, then $f^{-1}(p)$ is a Nash submanifold. A sphere, for instance, is thus Nash.

The next lemma is crucial in our paper; see [Shiota 1987, p. 44].

Lemma 2. Let $X$ be a closed semialgebraic subset of $\mathbb{R}^{n}$ of dimension $d$. If there is an open set $U$ of $X$ such that $U$ is a $C^{\infty}$ submanifold of dimension $d$ in $\mathbb{R}^{n}$, then around each point $p$ of $U$ there is an open neighborhood $B \subset U$ that is a semialgebraic subset of $\mathbb{R}^{n}$.

\section{Proper Dupin hypersurfaces are algebraic}

Let $f: M^{n-1} \rightarrow \mathbb{R}^{n}$ be a proper Dupin hypersurface. Suppose $f$ has $g$ principal curvatures $\kappa_{1}, \ldots, \kappa_{g}$, with multiplicities $m_{1}, \ldots, m_{g}$. Let $\mathscr{A}_{1}, \ldots, \mathscr{A}_{g}$ be a partition of the set $\{1, \ldots, n-1\}$ into disjoint subsets for which the cardinality of $\mathscr{A}_{i}$ is the multiplicity $m_{i}$ for $i=1, \ldots, g$.

For any point $p \in M$, the $i$-th curvature surface through $p$ is either an open subset of a sphere of dimension $m_{i}$ in $\mathbb{R}^{n}$ or an open subset of a plane of dimension $m_{i}$; see [Cecil and Ryan 1985]. Inversion in a sphere whose center does not lie on the union of all these spheres and planes will transform all curvature surfaces of $f(M)$ to open subsets of spheres. Thus, we may assume that all curvature surfaces of $f$ are open subsets of spheres of the appropriate dimensions.

Denote the center of the sphere of dimension $m_{i}$ containing the $i$-th curvature surface through $f(p)$ by $c_{i}(p) \in \mathbb{R}^{n}$, and denote its radius by $r_{i}(p)=\left|f(p)-c_{i}(p)\right|$. It is well known that $c_{i}(p)$ is rationally determined by $j_{p}^{3}(f)$, the 3-jet of $f$ at $p$; see [Cecil and Ryan 1985, p. 140-147] and [Ryan 1969]. If $e_{a}(p)$ for $a \in \mathscr{A}_{i}$ form 
an orthonormal basis of principal vectors for the $i$-th principal curvature, then they are determined by $j_{p}^{2}(f)$, the 2-jet of $f$ at $p$.

Since the sphere is Nash, for any point $p \in M$ there is an analytic algebraic parametrization of an open subset about $f(p)$ of the $i$-th curvature surface through $f(p)$ given by

$$
\mathbf{x}\left(s_{i}\right)=c_{i}(p)+s_{i}^{0}\left(s_{i}\right)\left(f(p)-c_{i}(p)\right)+r_{i}(p) \sum_{a \in \mathscr{A}_{i}} s_{i}^{a} e_{a}(p),
$$

where $s_{i}^{0}\left(s_{i}\right)=\sqrt{1-\left|s_{i}\right|^{2}}$ for all $s_{i}$ in the open ball $B^{m_{i}}\left(\epsilon_{i}\right) \subset \mathbb{R}^{m_{i}}$ for some radius $\epsilon_{i}$ satisfying $0<\epsilon_{i}<1$. The components of $s_{i}$ are denoted $s_{i}^{a}$ for each $a \in \mathscr{A}_{i}$, so that $\left|s_{i}\right|^{2}=1-\sum_{a \in \mathscr{A}_{i}}\left(s_{i}^{a}\right)^{2}$. The vectors $c_{i}(p), f(p)$, and $e_{a}(p)$ are determined by $j_{p}^{3}(f)$ for all $a \in \mathscr{A}_{i}$,

Lemma 3. For any $s_{i} \in B^{m_{i}}\left(\epsilon_{i}\right)$ in the parametrization (3-1), $j_{x_{i}\left(s_{i}\right)}^{k}(f)$ depends analytically algebraically on $s_{i}$ and on $j_{p}^{k+3}(f)$ for any $k \geq 0$, where $x_{i}\left(s_{i}\right)$ denotes the point for which $f\left(x_{i}\left(s_{i}\right)\right)=\mathbf{x}\left(s_{i}\right)$.

Proof. For some $\delta>0$, there exists $y: B^{n-1-m_{i}}(\delta) \rightarrow Y \subset M$, an embedded submanifold through $y(0)=p$ transverse to the $i$-th curvature surface through each of its points. Let $c_{i}(y)$ be the center of the $i$-th curvature surface through $f(y)$. Let $e_{j}$ for $j=1, \ldots, n-1$ be a smooth orthonormal frame field of principal vectors on a neighborhood of $p$. For each $y \in Y$,

$$
f\left(x_{i}\left(s_{i}\right), y\right)=c_{i}(y)+s_{i}^{0}\left(s_{i}\right)\left(f(y)-c_{i}(y)\right)+r_{i}(y) \sum_{a \in \mathscr{A}_{i}} s_{i}^{a} e_{a}(y)
$$

is a parametrization of a neighborhood of the $i$-th curvature surface through $f(y)$. Thus, for $\epsilon_{i}$ and $\delta$ sufficiently small, $\left(x_{i}, y\right)$ is a parametrization of a neighborhood of $p$ in $M$.

From (3-2) we see that the partial derivatives of $f$ with respect to the $s_{i}$ variables at $\left(s_{i}, y\right)$ depend on $j_{(0, y)}^{3}(f)$ and analytically algebraically on $s_{i}$, since all partial derivatives of $s_{i}^{0}\left(s_{i}\right)$ are analytic algebraic. Again from (3-2) we see that the partial derivatives of $f$ with respect to the $y$ variables at $\left(s_{i}, y\right)$ depend analytically algebraically on $s_{i}$ and on

$$
j_{(0, y)}^{1}\left(c_{i}\right), \quad j_{(0, y)}^{1}(f), \quad j_{(0, y)}^{1}\left(r_{i}\right), \quad j_{(0, y)}^{1}\left(e_{a}\right) \quad \text { for all } a \in \mathscr{A}_{i} .
$$

Since $c_{i}(0, y), f(0, y), r_{i}(0, y)$, and the $e_{a}(0, y)$ are determined by $j_{(0, y)}^{3}(f)$, it follows that their 1 -jets are determined by

$$
j_{(0, y)}^{1}\left(j^{3}(f)\right)=j_{(0, y)}^{4}(f) .
$$


Taking higher derivatives of (3-2) in this way, we see that $j_{\left(x_{i}\left(s_{i}\right), p\right)}^{k}(f)$ depends analytically algebraically on $s_{i}$ and on

$$
j_{p}^{k}\left(j^{3}(f)\right)=j_{p}^{k+3}(f) .
$$

Theorem 4. For any point $p \in M$, there exists an analytic algebraic parametrization of a neighborhood of $f(p)$ in $f(M) \subset \mathbb{R}^{n}$, where analytic algebraic means that the component functions are analytic algebraic. This collection of analytic algebraic parametrizations defines an analytic structure on $M$ with respect to which $f$ is analytic. In addition, it shows that any point $p \in M$ has an open neighborhood $U \subset M$ such that $f(U)$ is a semialgebraic subset of $\mathbb{R}^{n}$.

Proof. The idea is to iterate parametrizations along curvature surfaces, starting with the first. Choose a point $p \in M$. Setting $i=1$ in Equation (3-1) gives an analytic algebraic parametrization of a neighborhood of $f(p)$ of the first curvature surface through $p$. To simplify the following notation, we will write $f\left(s_{1}\right)$ in place of $f\left(x_{1}\left(s_{1}\right)\right)$, and likewise $c_{2}\left(s_{1}\right), r_{2}\left(s_{1}\right)$, and $e_{b}\left(s_{1}\right)$ without showing explicitly the composition with the map $x_{1}\left(s_{1}\right)$, et cetera.

For each $s_{1}$, parametrize a neighborhood of the second curvature surface through $f\left(s_{1}\right)$ by

$$
f\left(s_{1}, s_{2}\right)=c_{2}\left(s_{1}\right)+s_{2}^{0}\left(s_{2}\right)\left(f\left(s_{1}\right)-c_{2}\left(s_{1}\right)\right)+r_{2}\left(s_{1}\right) \sum_{b \in \mathscr{A}_{2}} s_{2}^{b} e_{b}\left(s_{1}\right),
$$

where $c_{2}\left(s_{1}\right), f\left(s_{1}\right)$, and $e_{b}\left(s_{1}\right)$ for $b \in \mathscr{A}_{2}$ are determined by $j_{s_{1}}^{3}(f)$, which in turn depends analytically algebraically on $s_{1}$ and $j_{p}^{3}\left(j^{3}(f)\right)=j_{p}^{6}(f)$, by Lemma 3 .

If $g>2$, then one more step should make the iteration clear. For each $s_{1}, s_{2} \in$ $B_{1}\left(\epsilon_{1}\right) \times B_{2}\left(\epsilon_{2}\right)$, parametrize a neighborhood of the third curvature surface through $f\left(s_{1}, s_{2}\right)$ by

$$
\begin{aligned}
f\left(s_{1}, s_{2}, s_{3}\right)=c_{3}\left(s_{1}, s_{2}\right)+s_{3}^{0}\left(s_{3}\right)\left(f\left(s_{1}, s_{2}\right)-c_{3}\left(s_{1}, s_{2}\right)\right) & \\
& +r_{3}\left(s_{1}, s_{2}\right) \sum_{c \in \mathscr{A}_{3}} s_{3}^{c} e_{c}\left(s_{1}, s_{2}\right) .
\end{aligned}
$$

Now $j_{\left(s_{1}, s_{2}\right)}^{3}(f)$, which determines $c_{3}\left(s_{1}, s_{2}\right), f\left(s_{1}, s_{2}\right)$, and $e_{c}\left(s_{1}, s_{2}\right)$, itself depends analytically algebraically on $s_{2}$ and $j_{\left(s_{1}, 0\right)}^{3}\left(j^{3}(f)\right)=j_{\left(s_{1}, 0\right)}^{6}(f)$, which likewise depends on $s_{1}$ and $j_{p}^{3}\left(j^{6}(f)\right)=j_{p}^{9}(f)$. Continuing in this way, we parametrize a neighborhood of the $g$-th curvature surface through $f\left(s_{1}, \ldots, s_{g-1}\right)$ by

$$
\begin{aligned}
f\left(s_{1}, \ldots, s_{g}\right)= & c_{g}\left(s_{1}, \ldots, s_{g-1}\right) \\
& +s_{g}^{0}\left(s_{g}\right)\left(f\left(s_{1}, \ldots, s_{g-1}\right)-c_{g}\left(s_{1}, \ldots, s_{g-1}\right)\right) \\
& +r_{g}\left(s_{1}, \ldots, s_{g-1}\right) \sum_{d \in A_{g}} s_{g}^{d} e_{d}\left(s_{1}, \ldots, s_{g-1}\right),
\end{aligned}
$$


which is analytic algebraic in $\left(s_{1}, \ldots, s_{g}\right) \in B^{m_{1}}\left(\epsilon_{1}\right) \times \cdots \times B^{m_{g}}\left(\epsilon_{g}\right)$ and depends on the finite set of constants determined by $j_{p}^{3 g}(f)$. By a standard argument [Thorbergsson 1983, p. 497], $f\left(s_{1}, \ldots, s_{g}\right)$ parametrizes a neighborhood of $p \in M$. Finally, since a finite product of open balls is a semialgebraic subset of $\mathbb{R}^{n-1}$, its image under the analytic algebraic map (3-3) is a semialgebraic subset of $\mathbb{R}^{n}$.

Corollary 5. A connected proper Dupin hypersurface $M$ in $\mathbb{R}^{n}$ is an open subset of a connected component of the irreducible algebraic set $\bar{M}^{\text {zar }}$ of dimension $n-1$.

Proof. By the theorem, for any point $p \in M$, there is an open neighborhood $U \subset M$ of the point that is a semialgebraic subset of $\mathbb{R}^{n}$. The result now follows from Lemma 1.

A slightly more general result holds that we shall apply to Dupin hypersurfaces.

Corollary 6. If a connected analytic hypersurface $N \subset \mathbb{R}^{n}$ contains a connected proper Dupin hypersurface $M$, then $N$ is an open subset of the connected component of an irreducible algebraic set that contains $M$.

Proof. An open subset of $M$ is open in $N$; so the result follows from Lemma 1.

\section{The finiteness condition}

A connected Dupin hypersurface $M$ in $\mathbb{R}^{n}$ has an open dense subset $\mathscr{G}$ such that each connected component of $\mathscr{G}$ is proper Dupin. In fact, $\mathscr{G}$ is the set on which the multiplicities of the principal curvatures are locally constant; see [Singley 1975]. Decompose $\mathscr{G}$ into its at most countably many disjoint connected components $\mathscr{G}_{1}$, $\mathscr{G}_{2}, \mathscr{G}_{3}, \ldots$ By Corollary 6 , if the Dupin hypersurface $M$ were an analytic submanifold of $\mathbb{R}^{n}$, then, since it contains the connected proper Dupin hypersurface $\mathscr{G}_{1}$, it would be contained in an irreducible algebraic set of dimension $n-1$. Hence, our goal is to prove that $M$ is an analytic hypersurface of $\mathbb{R}^{n}$.

Let $\mathscr{G}^{c}$ denote the complement of $\mathscr{G}$ in $M$. It is the set of points in $M$ that do not have a proper Dupin neighborhood.

Definition 7. A connected Dupin hypersurface $M$ of $\mathbb{R}^{n}$ has the local finiteness property if there is a subset $S \subset \mathscr{G}^{c}$ that is closed in $M$ such that $S$ disconnects $M$ into only a finite number of connected components, and for each point $p \in \mathscr{G}^{c}$ not in $S$, there is an open neighborhood $W$ of $p$ in $M$ such that $W \cap \mathscr{G}$ contains a finite number of connected open sets whose union is dense in $W$. We call $S$ the set of bad points in $\varphi^{c}$.

Theorem 8. Let $M$ be a connected Dupin hypersurface in $\mathbb{R}^{n}$. If $M$ has the local finiteness property, then it is an analytic submanifold of $\mathbb{R}^{n}$ and is therefore contained in a connected component of dimension $n-1$ of an irreducible algebraic set. 
Proof. Let $\mathscr{G} \subset M$ be the open dense subset of $M$ whose connected components are proper Dupin hypersurfaces in $\mathbb{R}^{n}$. Let $S \subset \mathscr{G}^{c}$ be the set of bad points in $\mathscr{G}^{c}$. By the local finiteness property, each point $p \in \mathscr{G}^{c}$ not in $S$ has a neighborhood $W$ that is open in $M$ such that $W \cap \mathscr{G}$ contains a finite number of connected open sets $U_{1}, \ldots, U_{s}$ whose union is dense in $W$. Note that $G$ being dense in $M$ implies that $W \subset \bigcup_{1}^{s} \bar{U}_{i}$, where $\bar{U}_{i}$ is the topological closure of $U_{i}$ in $M$. By Corollary 6, each $U_{i}$ is contained in an irreducible algebraic set $C_{i}$. Then $\bar{U}_{i}$ is a subset of $C_{i}$ since $C_{i}$ is closed. Hence, $W \subset \bigcup_{1}^{s} \bar{U}_{i} \subset \bigcup_{1}^{s} C_{i}$, which is a semialgebraic subset of $\mathbb{R}^{n}$.

By Lemma 2 , since $W$ is a $C^{\infty}$ manifold, there is a connected open semialgebraic subset $U$ of $\bigcup_{1}^{s} C_{i}$ contained in $W$ with $p \in U$. The intersection $B$ of $U$ with any open ball of $\mathbb{R}^{n}$ centered at $p$ is still an open subset of $M$ and a semialgebraic subset. Thus, we may assume $B$ is so small that it is the graph of $h: D \rightarrow \mathbb{R}$ for some open set $D \subset \mathbb{R}^{n-1}$ by performing a linear change of coordinates $\left(x^{1}, \ldots, x^{n}\right)$ in $\mathbb{R}^{n}$ if necessary. Since the projection $\pi:\left(x^{1}, \ldots, x^{n}\right) \mapsto\left(x^{1}, \ldots, x^{n-1}\right)$, which is semialgebraic, sends $B$ to $D$, we see $D$ is a semialgebraic subset of $\mathbb{R}^{n-1}$. Thus the map $h$ is semialgebraic, because its graph $\{(x, h(x)): x \in D\}=B$ is a semialgebraic subset of $\mathbb{R}^{n}$. Therefore $h$ is a Nash function, and so it must be analytic algebraic, and the map $H: D \rightarrow B, H(x)=(x, h(x))$ is an analytic algebraic parametrization of the open neighborhood $B \subset M$ about $p$. Since $p \in \mathscr{G}^{c} \backslash S$ was arbitrary, and since every point of $\mathscr{G}$ has a neighborhood with an analytic algebraic parametrization by Theorem 4, it follows that $M \backslash S$ is an analytic submanifold of $\mathbb{R}^{n}$. Since $M \backslash S$ has a finite number of connected components, each of which contains an open subset that is a semialgebraic subset of $\mathbb{R}^{n}$, it follows that $M \backslash S$ is contained in the union $C$ of finitely many irreducible algebraic sets. Then $M$ is contained in the topological closure of $M \backslash S$, which in turn must be contained in the closed set $C$.

Now let $q$ be any point in $S$ and consider $M$ itself as an open neighborhood about $q$. Then $M$ is a $C^{\infty}$ manifold contained in the semialgebraic set $C$. Apply the above argument to conclude that some open neighborhood of $q$ in $M$ is the image of an analytic algebraic parametrization. Hence, every point of $M$ has an analytic parametrization, and $M$ is an analytic hypersurface of $\mathbb{R}^{n}$.

Theorem 9. (a) If $M^{n-1}$ is a connected, compact taut hypersurface in $\mathbb{R}^{n}$ that satisfies the local finiteness property, then $M^{n-1}$ is an analytic submanifold and a connected component of an irreducible algebraic subset of $\mathbb{R}^{n}$.

(b) If $M^{m}$ is a connected, compact taut submanifold of codimension greater than one in $\mathbb{R}^{n}$ such that the tube $M_{\epsilon}$ over $M$, which is Dupin, satisfies the local finiteness property, then $M$ is an analytic submanifold and a connected component of an irreducible algebraic subset of $\mathbb{R}^{n}$.

Proof. (a) Since a taut hypersurface must be Dupin [Pinkall 1986], this follows immediately from Theorem 8. 
For (b), let $M$ be an embedded taut connected, compact submanifold in $\mathbb{R}^{n}$ and let $M_{\epsilon}$ be a tube over $M$ of sufficiently small radius that $M_{\epsilon}$ is an embedded hypersurface in $\mathbb{R}^{n}$. Then $M_{\epsilon}$ is a Dupin hypersurface; see [Pinkall 1986]. Thus $M_{\epsilon} \subset \mathbb{R}^{n}$ is semialgebraic by Theorem 8 , since $M_{\epsilon}$ satisfies the local finiteness property. Then $M$ itself is algebraic, since it is the image of the semialgebraic focal map $F_{\epsilon}: M_{\epsilon} \rightarrow M \subset \mathbb{R}^{n}$ given by $F_{\epsilon}(x)=x-\epsilon \xi$, where $\xi$ is the outward field of unit normals to the tube $M_{\epsilon}$.

\section{Ends and Alexander cohomology}

An end of a noncompact manifold $X$ is an equivalence class of sequences of connected open neighborhoods $X, U_{1}, U_{2}, \ldots$, where $U_{n+1} \subset U_{n}$, such that the intersection of the closures of these sets is the empty set, subject to the equivalence relation $\left(X, U_{1}, U_{2}, \ldots\right) \sim\left(X, W_{1}, W_{2}, \ldots\right)$ if for each $U_{i}$ there is a $j$ with $U_{i} \subset W_{j}$ and for each $W_{k}$ there is an $m$ such that $W_{k} \subset U_{m}$; see [Hughes and Ranicki 1996].

Let $S$ be a closed subset of a manifold $M$. The $k$-th Alexander cohomology group is defined to be the direct limit $\bar{H}^{k}(S):=\lim _{\rightarrow} H^{k}(V)$, where $H^{k}(V)$ is the usual $k$-th singular cohomology group of open $V \supset S$.

For any topological space $X$, a function $f$ from $X$ to $\mathbb{Z}_{2}$ is said to be locally constant if there is an open covering $U$ of $X$ such that $f$ is constant on each element of $U$. This theorem can be found in [Spanier 1966, p. 309]:

Theorem 10. $\bar{H}^{0}(S)$ is the $\mathbb{Z}_{2}$-module of locally constant functions from $S$ to $\mathbb{Z}_{2}$. In particular, if all the topological connected components of $S$ are open in $S$, then the rank of $\bar{H}^{0}(S)$ is identified with the cardinality of the set of connected components of $S$.

We only sketch the proofs of the following two corollaries of this theorem.

Corollary 11. Let $M$ be a compact simply connected manifold. Let $S \subset M$ be a closed subset for which $M \backslash S$ has only finitely many connected components. Suppose all the connected components of $S$ are open (whose cardinality is necessarily finite) in $S$. Then the number of ends of $M \backslash S$ is finite.

That $M$ is simply connected, so that $H^{1}(M)=0$, is employed for the short exactness of the $H^{0}$-block of a Mayer-Vietoris sequence obtained by the covering of $M$ by $M \backslash S$ and $U_{j}$ for $j=1,2, \ldots$, a sequence of shrinking neighborhoods of $S$ corresponding to the ends of $M \backslash S$. This gives a counting of the rank of $H^{0}\left(U_{j}\right)$, which would diverge to infinity if the number of ends of $M \backslash S$ were infinite. But then the preceding theorem would yield a contradiction when one takes the direct limit over $U_{j}$. 
Corollary 12. Let $M$ be a manifold of finitely many connected components, and let $S \subset M$ be closed. If $M \backslash S$ has finitely many ends, then $S$ has finitely many connected components each of which is open in $S$.

For the proof, one chases the diagram of the Mayer-Vietoris sequence up to $H^{1}(M)$ to show the number of connected components of $U_{j}$ is bounded, and so eventually it stabilizes to a number $m$. From this it follows that $\bar{H}^{0}(S)$ is of rank $m$ by taking direct limit over $U_{j}$, and $U_{j} \cap S$ for large $j$ constitute the $m$ open connected components of $S$. We call $S$ the end set of $M \backslash S$.

\section{Application to compact taut submanifolds}

Let $M$ be a compact taut hypersurface in $S^{n}$. Consider the normal exponential map $E: M \times(-\pi, \pi) \rightarrow S^{n}$, where

$$
E:(p, t) \mapsto \cos (t) p+\sin (t) \mathbf{n},
$$

with $\mathbf{n}$ the chosen unit normal field of $M$. Here, $E$ is smooth at points where $t=-\pi, \pi$.

A point $q=E(p, t)$ is called a focal point of multiplicity $m>0$ of $M$ at $p$ if the nullity of the derivative $E_{*}$ is equal to $m$ at $(p, t)$. The set of all focal points is the focal set $\mathscr{F}$ of $M$. The focal points at $p$ are antipodally symmetric on the circle $E(p, t)$ with each principal curvature of the form $\cot (t)$ for some $t \neq 0$. Let $\mathscr{L}$ be the complement of $\mathscr{F}$ in $S^{n}$.

Lemma 13. $\mathscr{L}$ is connected in $S^{n}$.

Proof. By Federer's version of Sard's theorem [Federer 1969, p. 316], the image of the critical points of a given smooth function $f: \mathbb{R}^{t} \rightarrow \mathbb{R}^{s}$, at which the rank of the derivative is less than or equal to $v$, is of $\mathscr{H}^{v}$-measure 0 , where $\mathscr{H}^{v}$ denotes the Hausdorff $v$-dimensional measure.

Label the principal curvature functions by $\lambda_{1} \leq \lambda_{2} \leq \cdots \leq \lambda_{n-1}$. It is known that $\lambda_{j}$ are continuous; see [Ryan 1969]. Let $\cot \left(t_{j}\right):=\lambda_{j}$ for $0<t_{j}<\pi$. We know that the focal maps

$$
f_{j}(p)=\cos \left(t_{j}\right) p+\sin \left(t_{j}\right) \mathbf{n}
$$

are continuous and are smooth on a dense open set.

For each $i$, let $O_{i}$ be the open subset of $M$ on which $\lambda_{i}$ has multiplicity 1. ( $O_{i}$ could be empty.) Then $O_{i}$ consists of countably many open components $O_{i j}$ for $j=1,2, \ldots$ such that the restriction of $f_{i}$ to $\left(O_{i j}\right)$ is an embedded submanifold of dimension $n-2$. This follows from the fact that $\lambda_{i}$ is constant on its lines of curvature on each $O_{i j}$ by the Dupin condition; see [Cecil and Ryan 1985, p. 145].

Let $Z_{i}$ be the complement of $O_{i}$ in $M$. ( $Z_{i}$ could be empty.) At each point $p$ of $Z_{i}$, the principal curvature $\cot \left(t_{i}\right)$ must have multiplicity at least 2 , and so the 
normal exponential map $E$ has rank $\leq n-2$ at the point $\left(p, t_{i}\right)$. Thus the focal point $f_{i}(p)$ lies in the singular value set $K$ of points for which the derivative of $E$ has rank $\leq n-2$.

We conclude the entire focal set $\mathscr{F}$ is composed of the countably many embedded submanifolds $f_{i}\left(O_{i j}\right)$ of dimension $n-2$, their antipodal sets, and the set $K$, which has Hausdorff ( $n-2)$-measure zero by Federer's theorem quoted above. Therefore the Hausdorff $(n-1)$-measure of the whole focal set $\mathscr{F}$ is zero, which implies that the complement $\mathscr{E}$ of $\mathscr{F}$ is connected [Schoen and Yau 1994, p. 269].

Away from $\mathscr{F}$, the map $E$ is a local diffeomorphism. For each $0 \leq m \leq n-1$, we let $W_{m}$ be the set of points $(p, t)$ in $E^{-1}(\mathscr{L})$ for which the spherical distance function $d_{q}$, where $q=E(p, t)$, has index $m$ at $p$. Then the tautness of $M$ implies that $E: W_{m} \rightarrow \mathscr{E}$ is a degree $b_{m}$ (regular) covering map, where $b_{m}$ is the $m$-th Betti number. Therefore, by the connectedness of $\mathscr{E}$, the set $W_{m}$ decomposes into finitely many (connected) covering sheets $W_{m 1}, W_{m 2}, \ldots, W_{m s_{m}}$ onto $\mathscr{L}$, where $s_{m} \leq b_{m} . W_{m}$ will be composed of connected components either in $M \times(0, \pi)$ or $M \times(-\pi, 0)$. We denote by $W_{m j}^{+}$those $W_{m j}$ contained in $M \times(0, \pi)$. We set $W_{m}^{+}:=\bigcup_{j} W_{m j}^{+}$.

Lemma 14. All the connected components of $\mathscr{F}$ are open in $\mathscr{F}$. In particular, $\mathscr{L}$ has a finite number of ends.

Proof. Since each $f_{j}(M)$ for $1 \leq j \leq n-1$ defined in (6-1) is compact and pathconnected in $S^{n}$, we can group them and the sets antipodal to them into classes where the union of those $f_{j}(M)$ in each class is path-connected whereas the unions from different classes are disjoint. Call these disjoint unions $X_{1}, \ldots, X_{r}$, each of which, being a finite union of compact sets, is closed in $S^{n}$ and path-connected. Hence by the Urysohn separation lemma, there are disjoint open sets $O_{1}, \ldots, O_{r}$ that contain $X_{1}, \ldots, X_{r}$, respectively. This means that each (topological) component, now being just a path-connected component, is open in the relative topology. The conclusion follows by Corollary 11 .

Corollary 15. Each $W_{m j}^{+}$has a finite number of ends.

Proof. This follows since $E: W_{m j}^{+} \rightarrow \mathscr{L}$ is a proper map because it is a covering map of finite degree and because $\mathscr{L}$ has a finite number of ends.

Let pr: $M \times(-\pi, \pi) \rightarrow M$ be the projection. Since pr is an open map, the sets

$$
U_{m j}^{+}:=\operatorname{pr}\left(W_{m j}^{+}\right)
$$

are open and connected in $M$. We also set

$$
U_{m}^{+}:=\bigcup_{j} U_{m j}^{+}=\operatorname{pr}\left(W_{m}^{+}\right) .
$$


It is clear that $W_{m}^{-}$and $U_{m}^{-}$can be similarly defined on $M \times(-\pi, 0)$.

Definition 16. We define $\left(U_{m}^{*}\right)^{+}$to be the collection of all $x$ for which there exists a $t>0$ such that $(x, t)$ is a regular point of the normal exponential map $E$ and such that the spherical distance function $d_{y}$, where $y=E(x, t)$, has index $m$ at $x$.

Note that $U_{m}^{+} \subset\left(U_{m}^{*}\right)^{+}$by definition. Further, any point $p$ in the complement $\left(U_{m}^{*}\right)^{+} \backslash U_{m}^{+}$satisfies the condition that every $q=E(p, t)$ for $t>0$ on the normal exponential circle, such that $d_{q}$ has a nondegenerate critical point of index $m$ at $p$, is a focal point of some other point in $M$.

Lemma 17. $\left(U_{m}^{*}\right)^{+}$is open in $M$.

Proof. This follows from the property that $E$ is a local diffeomorphism around $(x, t)$ for $t>0$, so that $E(x, t)$ is nonfocal along the normal exponential circle. Hence, for a point $\left(x^{\prime}, t^{\prime}\right)$ near $(x, t)$, the point $E\left(x^{\prime}, t^{\prime}\right)$ is also nonfocal along the respective normal exponential circle. Now, $d_{y}$ has index $m$ at the nondegenerate critical point $x$. When $x^{\prime}$ is sufficiently close to $x$, the function $d_{y^{\prime}}$, where $y^{\prime}=E\left(x^{\prime}, t^{\prime}\right)$, is a slight perturbation of $d_{y}$. Since $x^{\prime}$ is a nondegenerate critical point of $d_{y^{\prime}}$ and since nondegenerate critical points are locally structurally stable, $d_{y^{\prime}}$ must also have index $m$ at $x^{\prime}$, and thus $x^{\prime} \in\left(U_{m}^{*}\right)^{+}$.

For $\left(p, t_{1}\right)$ and $\left(p, t_{2}\right)$ in $M \times(0, \pi)$, we say $\left(p, t_{1}\right)$ is equivalent to $\left(p, t_{2}\right)$ and write $\left(p, t_{1}\right) \sim\left(p, t_{2}\right)$ if the distance functions $d_{q_{1}}$ for $q_{1}=E\left(p, t_{1}\right)$ and $d_{q_{2}}$ for $q_{2}=E\left(p, t_{2}\right)$ have nondegenerate critical points of the same index $m$ at $p$. We then let $L_{m j}$ be this (trivial) line bundle over $U_{m j}^{+}$:

$$
L_{m j}:=\left\{(p, t) \in M \times(0, \pi):(p, t) \sim\left(p, t_{0}\right) \text { for some }\left(p, t_{0}\right) \in W_{m j}\right\} .
$$

Lemma 18. $L_{m j} \backslash W_{m j}^{+}$is of Hausdorff codimension at least 2 in $L_{m j}$. In particular, $L_{m j}$ and $U_{m j}^{+}$have a finite number of ends.

Proof. By the openness of $W_{m j}^{+}$, we know $L_{m j} \backslash W_{m j}^{+}$is closed in $L_{m j}$. We next show that $L_{m j} \backslash W_{m j}^{+}$is of Hausdorff codimension at least 2. Consider the restriction of the map $E$ on $L_{m j} \backslash W_{m j}^{+}$given by

$$
f:=(p, t) \in L_{m j} \backslash W_{m j}^{+} \mapsto E(p, t) \in \mathscr{F} .
$$

Note that $f$ is a finite-to-one map. This is because for any $z \in \mathscr{F}$, the height function $\ell_{z}$ is a perfect Morse-Bott function [Ozawa 1986], and each $p$, for which some point of the form $(p, t)$ is in $f^{-1}(z)$, is a nondegenerate critical point of index $m$ of $\ell_{z}$ on the taut hypersurface $M$. Such a nondegenerate critical point is a critical submanifold. Hence the total number of such points is no more than $b_{m}$, the $m$-th Betti number of $M$.

The image of $f$ is a closed subset of $\mathscr{F}$ of Hausdorff codimension at least 2 by Lemma 13. $E$ is a local diffeomorphism at $(p, t) \in L_{m j}$ when it is restricted to $L_{m j}$. 
Hence, there is a neighborhood $Y_{(p, t)} \subset L_{m j}$ around each $(p, t) \in L_{m j} \backslash W_{m j}^{+}$such that $f$ restricted to $Y_{(p, t)} \cap\left(L_{m j} \backslash W_{m j}^{+}\right)$is a homeomorphism into $\mathscr{F}$. It follows that the Hausdorff $(n-2)$-measure of $L_{m j} \backslash W_{m j}^{+}$is at most $b_{m}$ times that of $\mathscr{F}$, which is null in the ambient sphere $S^{n}$. So $L_{m j} \backslash W_{m j}^{+}$is of Hausdorff codimension at least 2 in $L_{m j}$. In particular, $L_{m j} \backslash W_{m j}^{+}$does not disconnect $L_{m j}$.

We now apply Corollary 12 with $S=L_{m j} \backslash W_{m j}^{+}, M=L_{m j}$, and $X=M \backslash S=W_{m j}^{+}$. Since $X$ has a finite number of ends, it follows that $S$ has finitely many components all of which are open in $S$. Now, suppose $M$ has infinitely many ends. Then there is a decreasing sequence of open sets $X_{1}, X_{2}, \ldots$ in $X$ such that $X \backslash X_{j}$ is compact, $\bigcap_{j} \bar{X}_{j}=\varnothing$, and the number of unbounded connected components of $X_{j}$ increasingly diverges to infinity. As $S$ has finitely many components $S_{1}, \ldots, S_{t}$, all of which have Hausdorff codimension at least $2, S_{1}, \ldots, S_{t}$ cannot disconnect the connected components of $X_{j}$. We see $X_{j} \backslash S$ form a decreasing sequence whose disconnected components diverge to infinity, so that $W_{m j}^{+}=M \backslash S$ will have infinitely many ends. This contradiction establishes that $L_{m j}$ has a finite number of ends. Since $L_{m j}$ is a trivial line bundle over $U_{m j}^{+}$, so that $L_{m j} \simeq U_{m j}^{+} \times \mathbb{R}$, it follows that $U_{m j}^{+}$has a finite number of ends.

Corollary 19. $W_{m j}^{+}$is disjoint from $W_{m l}^{+}$if and only if $U_{m j}^{+}$is disjoint from $U_{m l}^{+}$. In particular, $U_{m}^{+}$has finitely many ends.

Proof. The backward direction is clear. To prove the forward direction, suppose $p \in U_{m j}^{+} \cap U_{m l}^{+}$. Let $X:=U_{m j}^{+} \cup U_{m l}^{+}$, and let $Y:=W_{m j}^{+} \cup W_{m l}^{+}$. Since $X$ is open and connected, we can form the (trivial) line bundle $L$ over $X$, where $L$ consists of $(p, t)$ for $p \in X$ such that the distance function $d_{q}$ for $q=E(p, t)$ has a nondegenerate critical point of index $m$ at $p$. Then $L=L_{m j} \cup L_{m l}$ and $Y \subset L$ by construction. However, the analysis in Lemma 18 shows that $L \backslash Y$ is of Hausdorff codimension at least 2; therefore $Y$ is connected, and so $W_{m j}^{+}$and $W_{m l}^{+}$cannot be disjoint. Now that $U_{m}^{+}$is the finite disjoint union of all $U_{m j}^{+}$, each of which has finitely many ends, it follows that the same is true for $U_{m}^{+}$.

Corollary 20. $U_{m}^{+}$is dense in $\left(U_{m}^{*}\right)^{+}$. In particular, $\left(U_{m}^{*}\right)^{+}$has a finite number of connected components.

Proof. Suppose that $U_{m}^{+}$is not dense in $\left(U_{m}^{*}\right)^{+}$. Then there is an open set $X \subset$ $\left(U_{m}^{*}\right)^{+}$that is disjoint from $U_{m}^{+}$. Proceeding as in Corollary 19, let us introduce the line bundle $L$ over $X$, which consists of all points $(p, t)$ for $t>0$ in which $p \in X$ and $d_{q}$ for $q=E(p, t)$ has a nondegenerate critical point of index $m$ at $p$.

Every point $(p, t) \in L$ is mapped to $\mathscr{F}_{F}$ via the map $E$, since $p \in\left(U_{m}^{*}\right)^{+} \backslash U_{m}^{+}$. As we see in Lemma 18, the map $f$ of Lemma 18 on $L$ is finite-to-one and regular at every point of $L$. Thus, the image of $f$ contains an open set of dimension 
$n=\operatorname{dim} L$. This contradicts that the Hausdorff codimension of $\mathscr{F}$ is at least two. Thus $U_{m}^{+}$is dense in $\left(U_{m}^{*}\right)^{+}$.

In particular, $\left(U_{m}^{*}\right)^{+}$also has a finite number of connected components, because any of its components will contain at least one component of $U_{m}^{+}$.

We say a point $p$ is a good point in the taut hypersurface $M$ if the multiplicities of the principal curvatures are locally constant around $p$. We denote the set of good points by $\mathscr{G}$. We know $\mathscr{G}$ is open and dense in $M$.

Our convention is that we label the principal curvature functions by $\lambda_{1} \leq \lambda_{2} \leq$ $\cdots \leq \lambda_{n-1}$. When we say $\left(m_{1}, m_{2}, \ldots, m_{g}\right)$ is a given sequence of principal multiplicities, we mean that

$$
\lambda_{1}=\cdots=\lambda_{m_{1}} \quad \text { and } \quad \lambda_{m_{1}+1}=\cdots=\lambda_{m_{1}+m_{2}}, \ldots,
$$

so that $m_{i}$ is the multiplicity of the $i$-th largest distinct principal curvature.

Lemma 21. There is a dense open subset of $\mathscr{G}$ such that for any $p$ in the subset, there is a point $q \in \mathscr{Z}$ between any two focal points on the normal exponential circle $E(p, t)$ for $t>0$.

Proof. Suppose $X$ is a connected component of $\mathscr{G}$, and assume multiplicities $\left(m_{1}, m_{2}, \ldots, m_{\mu}\right)$. Let $\sigma_{s}=m_{1}+\cdots+m_{s}$ for $1 \leq s \leq \mu$. Then $X \subset\left(U_{\sigma_{s}}^{*}\right)^{+}$. Then Corollary 20 implies that $X \cap U_{\sigma_{s}}^{+}$is dense and open in $X$.

Definition 22. We let $\varphi^{\circ}$ be the largest dense open subset of $\mathscr{G}$ over which Lemma 21 is true.

Lemma 23. Let $g$ be the maximum number of distinct principal curvatures on $M$. Let $M:=\left(m_{1}, \ldots, m_{g}\right)$ be a given maximal sequence of multiplicities. Let $O_{\mu} \subset \varphi^{\circ}$ be the (open) subset of $\varphi^{\circ}$ attaining these multiplicities. Let $\sigma_{j}=m_{1}+\cdots+m_{j}$ for $1 \leq j \leq g$. Then $O_{\mu}$ is the intersection of all the $U_{\sigma_{j}}^{+}$.

Proof. The intersection consists of all points $p$ such that there is some $0<t_{j}<\pi$ for which $p$ is of index $\sigma_{j}$ with respect to the distance function $d_{q}$, with $q=$ $E\left(p, t_{j}\right)$ for each $j$. So $p$ must have $g$ principal curvatures with multiplicities $m_{1}, m_{2}, \ldots, m_{g}$. The maximality of $g$ implies that $m_{j}$ cannot be broken further into smaller multiplicities. Also, since $p \in U_{\sigma_{j}}^{+}$, there is a $t$ for which $E(p, t)$ between the two appropriate focal points is in $\mathscr{E}$. We obtain $p \in \mathscr{G}^{\circ}$. So $\cap U_{\sigma_{j}}^{+} \subset \mathcal{O}_{M}$.

Conversely, since $0_{M} \subset \mathscr{G}^{\circ}$, the definition of $U_{\sigma_{j}}^{+}$implies that $\widehat{O}_{M} \subset \cap U_{\sigma_{j}}^{+}$.

We now treat the case of a taut submanifold of dimension 3 or 4 . First we prove a needed lemma in homology.

Lemma 24. Let $B_{1} \subset B_{2}$ be two closed disks. Then the $k$-th new topology of the taut submanifold $M$ added between $B_{1} \cap M$ and $B_{2} \cap M$ is the relative homology

$$
H_{k}\left(B_{2} \cap M, B_{1} \cap M\right) \text {. }
$$


Proof. This follows from the exact sequence

$$
\begin{aligned}
\longrightarrow H_{k}\left(B_{1} \cap M\right) \stackrel{i}{\longrightarrow} H_{k}\left(B_{2} \cap M\right) & \longrightarrow H_{k}\left(B_{2} \cap M, B_{1} \cap M\right) \\
& \stackrel{j}{\longrightarrow} H_{k-1}\left(B_{1} \cap M\right) \stackrel{i}{\longrightarrow} H_{k-1}\left(B_{2} \cap M\right) \longrightarrow
\end{aligned}
$$

and the fact that $i$ is injective by the injectivity property of tautness, so that $j$ is the zero map. It follows that

$$
H_{k}\left(B_{2} \cap M\right)=H_{k}\left(B_{1} \cap M\right) \oplus H_{k}\left(B_{2} \cap M, B_{1} \cap M\right),
$$

and hence the conclusion.

Corollary 25. If $B_{1} \subset B_{2} \subset B_{3}$ are three closed disks, then

$$
H_{k}\left(B_{3} \cap M, B_{1} \cap M\right)=H_{k}\left(B_{3} \cap M, B_{2} \cap M\right) \oplus H_{k}\left(B_{2} \cap M, B_{1} \cap M\right) .
$$

Therefore $H_{k}\left(B_{3} \cap M, B_{1} \cap M\right)$ is surjective to both $H_{k}\left(B_{3} \cap M, B_{2} \cap M\right)$ and $H_{k}\left(B_{2} \cap M, B_{1} \cap M\right)$.

Proof. Given three abelian groups $A \rightarrow B \rightarrow C$, where each arrow is an embedding, we have $C / B=(C / A) /(B / A)$.

Lemma 26. Suppose $\operatorname{dim} M=4$.

(a) If the maximum number of principal curvatures is $\geq 3$, then multiplicities $(1,3),(3,1)$, and $(2,2)$ cannot exist on open sets.

(b) If the maximum number of principal curvatures is 4, then the points with multiplicities $(1,1,2),(1,2,1)$, and $(2,1,1)$, at which the number of principal curvatures is not locally constant, cannot be approached by a sequence coming from an open set of points of the same multiplicities.

Proof. (a) Suppose the multiplicities are $(1,3)$ on an open set $O$. Let $x \in O$ and let $p$ be a boundary point of $O$. Let $c(t)$ be a smooth curve with $c(0)=x$ and $c(1)=p$. We can assume that $c(t) \in O$ for $0 \leq t<1$. Since $p$ is a boundary point of $O$, there must be a sequence of points with multiplicities $(1,1,2),(1,2,1)$, or $(1,1,1,1)$ that converges to $p$. The multiplicities must remain $(1,3)$ at $p$; otherwise, the list of multiplicities would drop to the single multiplicity (4), and this is impossible because a taut hypersurface with a single umbilic point must be a totally umbilic sphere; see [Cecil and Ryan 1985, p. 123]. We will handle the case where a sequence of points with multiplicities $(1,1,2)$ approaches $p$. The other cases are very similar.

At each point $c(t)$ for $0 \leq t<1$, the curvature surface $S(t)$ corresponding to the principal curvature $\lambda$ of multiplicity 3 is a 3 -dimensional metric sphere, which converges to the 3 -dimensional curvature surface, a 3 -sphere which corresponds to $\lambda$ at $p$, as $t$ approaches 1 . On the other hand, consider a sequence of points $\left\{y_{i}\right\}$ 
approaching $p$, where the multiplicities are $(1,1,2)$. For each $y_{i}$, the 2-dimensional curvature surface $C_{i}$ through $y_{i}$ corresponding to the principal curvature of multiplicity 2 is a topset for the spherical distance function centered at the corresponding focal point. Thus, by tautness, $C_{i}$ represents a nontrivial 2-dimensional homology class in $M$. As $y_{i}$ approaches $p$, these $C_{i}$ approach a 2-cycle $C$ in the curvature surface $S(1)$ that is nontrivial in the 2-dimensional homology of $S(1)$. This is a contradiction, since $S(1)$ is a 3 -sphere and has trivial 2-dimensional homology. A similar proof shows that multiplicities $(3,1)$ cannot exist on an open set.

Next suppose that the multiplicities are $(2,2)$ on an open set $O$. As in the argument above, let $x \in O$ and let $p$ be a boundary point of $O$. Let $c(t)$ be a smooth curve with $c(0)=x, c(1)=p$, and $c(t) \in O$ for $0 \leq t<1$. Then the multiplicities must be $(2,2)$ at $p$. Since $p$ is a boundary point of $O$, there must be a sequence of points with multiplicities $(1,1,2),(2,1,1)$, or $(1,1,1,1)$ that converges to $p$. We will handle the case where the multiplicities are $(1,1,2)$; the others are handled similarly. Then as in the argument above, at each point $c(t)$ for $0 \leq t<1$, the curvature surface $S(t)$ corresponding to the first principal curvature $\lambda$ of multiplicity 2 is a 2-dimensional metric sphere, which converges to the curvature surface, a 2-sphere, which corresponds to $\lambda$ at $p$. On the other hand, there is a sequence $\left\{y_{i}\right\}$ approaching $p$ such that the multiplicities are $(1,1,2)$ at $y_{i}$. Again, the topset property as above gives a contradiction.

For (b), in the case of multiplicities $(1,1,2)$ or $(2,1,1)$, the same type of argument takes care of this statement, since one can produce a point $p$ with the given multiplicities that is also a limit of a sequence of points with multiplicities $(1,1,1,1)$.

In the case of multiplicities $(1,2,1)$, we need to modify the argument slightly. As above, the nondegenerate critical manifold at $p$ corresponding to the principal curvature of multiplicity two is a metric 2 -sphere $S$, and we can also approach $p$ by a sequence of points with multiplicities $(1,1,1,1)$. However, in this case, the 1-dimensional circles approaching $p$ corresponding to the second and third multiplicities are not topsets. We can see that they still contribute in a nontrivial way to homology by the following argument.

At the point $p$, the multiplicities are $(1,2,1)$, and $p$ is a limit point of the open connected set $O$ on which the multiplicities are $(1,2,1)$. Let $q$ be the second focal point of $M$ at $p$ corresponding to the principal curvature of multiplicity two. As above, we can also approach $p$ by a sequence of points in the open set $U$ with multiplicities $(1,1,1,1)$. Thus near $p$, we can find points $x \in O$ and $y \in U$, with corresponding second focal points $u$ and $v$ near $q$ such that the height functions satisfy $\ell_{u}(x)=a$ and $\ell_{v}(y)=b$. We can choose $x$ and $y$ so close to $p$ that we can find positive numbers $\epsilon$ and $\delta$ for which

$$
M_{a-\epsilon}\left(\ell_{u}\right) \subset M_{b-\delta}^{-}\left(\ell_{v}\right) \subset M_{b}\left(\ell_{v}\right) \text { and } M_{a}\left(\ell_{u}\right) \subset M_{b+\delta}^{-}\left(\ell_{v}\right) \subset M_{a+\epsilon}\left(\ell_{u}\right) .
$$


Also, using a genericity argument, we can assume that the points $x$ and $y$ are chosen so that the critical submanifolds of the corresponding height functions $\ell_{u}$ and $\ell_{v}$ are at distinct levels, and we may choose $\epsilon$ and $\delta$ sufficiently small so that $a$ (respectively $b$ ) is the only critical value between $a-\epsilon$ and $a+\epsilon$ (respectively between $b-\delta$ and $b+\delta$ ).

Lemma 24 says that the new $k$-th homology between the levels $a-\epsilon$ and $a+\epsilon$ of $\ell_{u}$ is

$$
H_{k}\left(M_{a+\epsilon}\left(\ell_{u}\right), M_{a-\epsilon}\left(\ell_{u}\right)\right) .
$$

Corollary 25 says that the group in Equation (6-2) is surjective to

$$
H_{k}\left(M_{a+\epsilon}\left(\ell_{u}\right), M_{b-\delta}\left(\ell_{v}\right)\right),
$$

where in Lemma 24, we take

$$
B_{1} \cap M=M_{a-\epsilon}\left(\ell_{u}\right), \quad B_{2} \cap M=M_{b-\delta}\left(\ell_{v}\right), \quad B_{3} \cap M=M_{a+\epsilon}\left(\ell_{u}\right) .
$$

The same lemma says that the group in Equation (6-3) is surjective to

$$
H_{k}\left(M_{b+\delta}\left(\ell_{v}\right), M_{b-\delta}\left(\ell_{v}\right)\right),
$$

where

$$
B_{1} \cap M=M_{b-\delta}\left(\ell_{v}\right), \quad B_{2} \cap M=M_{b+\delta}\left(\ell_{v}\right), \quad B_{3} \cap M=M_{a+\epsilon}\left(\ell_{u}\right) .
$$

It follows from these considerations that the group in Equation (6-2) is surjective to the group in Equation (6-4). However, by Morse-Bott critical point theory [Dubrovin et al. 1990, p. 239], we have

$$
H_{k}\left(M_{a+\epsilon}\left(\ell_{u}\right), M_{a-\epsilon}\left(\ell_{u}\right)\right)=H_{k-\mu}(W),
$$

where $W$ is the critical manifold of $\ell_{u}$ at $x$ and $\mu$ is the index at $x$. Similarly, we have

$$
H_{k}\left(M_{b+\delta}\left(\ell_{v}\right), M_{b-\delta}\left(\ell_{v}\right)\right)=H_{k-v}(V),
$$

where $V$ is the critical submanifold of $\ell_{v}$ at $y$ and $v$ is the index at $y$.

Now the critical submanifold $W$ at $x$ is a 2-sphere of index $\mu=1$, whereas the critical submanifold $V$ at $y$ is a circle of index $v=1$. Using $k=2$ in equations (6-5) and (6-6), we see that $0=H_{1}\left(W, \mathbb{Z}_{2}\right)$ is surjective to $H_{1}\left(V, \mathbb{Z}_{2}\right)=\mathbb{Z}_{2}$. This is a contradiction.

We now prove that taut hypersurfaces of dimensions 3 and 4 are algebraic.

Theorem 27. Let $M$ be a compact taut hypersurface in $S^{n}$ that is not a hypersphere. If $\operatorname{dim} M=3$ or 4 , then $M$ is algebraic. 
Proof. Assume $\operatorname{dim} M=4$ and the maximum number of multiplicities is 4 . By Lemma 26, multiplicities $(1,3),(3,1)$, and $(2,2)$ cannot exist on open sets. Meanwhile, the set of points with multiplicities $(1,1,1,1)$ is open. Since by Lemma 26 a point with multiplicities $(1,1,2)$ (or $(1,2,1)$ or $(2,1,1))$ at which the number of principal curvatures is not locally constant cannot be approached by a sequence coming from an open set of points of the same multiplicities, we see that such points must be entirely surrounded by points with multiplicities $(1,1,1,1)$. As a result, an open set of points with multiplicities $(1,1,2)$ will approach boundary points with multiplicities either $(1,3)$ or $(2,2)$.

The set $\varphi$ of good points are those with multiplicities $(1,1,1,1)$ and the points with multiplicities $(1,1,2),(1,2,1)$, or $(2,1,1)$ that exist on open sets. $\mathscr{G}$ is open and dense in $M$. We let $S$ be the subset of $\varphi^{c}$ consisting of points of multiplicities $(1,3),(3,1)$, or $(2,2)$. We wish to confirm the local finiteness property.

Firstly, $S$ is closed in $M$. This is because any converging sequence of points of the indicated multiplicities must maintain the same type of multiplicities.

We next show that $M \backslash S$ has finitely many connected components. It comes down to showing that $A:=\left(U_{1}^{*}\right)^{+}$(respectively $B:=\left(U_{2}^{*}\right)^{+}$or $\left.C:=\left(U_{3}^{*}\right)^{+}\right)$ with points of multiplicities $(1,3)$ (respectively $(2,2)$ or $(3,1))$ removed has only finitely many connected components, for then the union of the three resulting sets is exactly $M \backslash S$, which, being a union, must have finitely many connected components. However, as before, this follows from the Mayer-Vietoris sequence applied to the open covers $A$ and $B \cup C$ of $M$, et cetera. Note that $A$ is composed of points with multiplicities $(1,1,1,1),(1,1,2),(1,2,1)$, or $(1,3)$; $B$ 's points have multiplicities $(1,1,1,1),(1,1,2),(2,1,1)$, or $(2,2)$; and $C$ 's points have multiplicities $(1,1,1,1),(1,2,1),(2,1,1)$, or $(3,1)$. Hence $A \cap(B \cup C)$ is exactly $A$ with points of multiplicities $(1,3)$ removed.

Lastly, we verify that each point in $\mathscr{G}^{c} \backslash S$ has a open neighborhood $W$ in $M$ such that $W \cap \mathscr{G}$ contains finitely many connected open sets whose union is dense in $W$.

As mentioned earlier, $G^{c} \backslash S$ is completely surrounded by points of multiplicities $(1,1,1,1)$. Each point $p \in \mathscr{G}^{c} \backslash S$ of multiplicities $(1,1,2)$ has a small neighborhood that contains no points of multiplicities $(1,2,1)$ or $(2,1,1)$, et cetera. In other words, the sets $T_{1}, T_{2}, T_{3}$ of points of multiplicities $(1,1,2),(1,2,1),(2,1,1)$, respectively, are contained in disjoint open sets $O_{1}, O_{2}, O_{3}$, respectively, where $O_{i} \backslash T_{i}$ for $1 \leq i \leq 3$ consists of only points of multiplicities $(1,1,1,1)$.

Let $X \subset\left(U_{3}\right)^{+}$be the subset of points of multiplicities $(1,1,1,1) . X$ is dense in the set of points of multiplicities $(1,1,1,1)$ by Lemmas 21 and 23 . It follows that $T_{1}$ is contained in the end sets of the finitely many end components $E_{1}, \ldots, E_{s}$ of $U_{3}^{+}$on which the multiplicities remain $(1,1,1,1)$. Let $W$ be the union of $O_{1}$ above and $E_{1}, \ldots, E_{s}$. ( $O_{1}$ is the union of neighborhoods of points of multiplicities 
$(1,1,2)$. We make sure each of these neighborhoods is so small that its intersection with $U_{3}^{+}$is contained in the end components $E_{1}, \ldots, E_{s}$.) Then $W \cap G_{\text {consists }}$ of only points of multiplicities $(1,1,1,1)$ and also contains open sets $E_{1}, \ldots, E_{s}$ whose union is dense in $W$.

Similarly, the same conclusion is true for points of multiplicities $(2,1,1)$ and $(1,2,1)$ in $g^{c}$ with $U_{3}^{+}$replaced by $U_{1}^{+}$and $U_{2}^{+}$, respectively. Hence $M$ is algebraic.

The case $\operatorname{dim} M=3$ and $\operatorname{dim} M=4$ when the maximal number of multiplicities is 3 follows from the same procedure. For the latter case, $\varphi^{c}$ is the set of points with multiplicities $(1,3),(3,1)$ or $(2,2)$, which coincides with $S$; thus the set $\varphi^{c} \backslash S=\varnothing$, and the local finiteness condition is automatically satisfied on $\varphi^{c} \backslash S$. Similarly for the former case, we have $\varphi^{c}=S$, which is the set of points with multiplicities $(1,2)$ or $(2,1)$.

By considering a tube $M_{\epsilon}$ with a small radius $\epsilon$ over a compact taut submanifold $M$, the method employed in the preceding theorem gives the theorem below in dimensions 3 and 4.

Banchoff [1970] showed that a taut compact 1-dimensional submanifold of $S^{n}$ must be a metric circle in $S^{n}$, which is certainly algebraic. In the same paper, he also showed that if $M$ is a taut compact 2-dimensional surface substantially embedded in $S^{n}$, then $M$ is a metric 2-sphere, a cyclide of Dupin in $S^{3}$, or a spherical Veronese surface $V \subset S^{4}$. All of these surfaces are algebraic. Thus, we have the following result.

Theorem 28. Let $M$ be a compact taut $m$-dimensional submanifold in $S^{n} \subset \mathbb{R}^{n+1}$. If $m \leq 4$, then $M$ is algebraic.

\section{References}

[Banchoff 1970] T. F. Banchoff, "The spherical two-piece property and tight surfaces in spheres", $J$. Differential Geometry 4 (1970), 193-205. MR 42 \#3720 Zbl 0208.49502

[Bochnak et al. 1998] J. Bochnak, M. Coste, and M.-F. Roy, Real algebraic geometry, vol. 36, Ergebnisse der Mathematik, Springer, Berlin, 1998. MR 2000a:14067 Zbl 0284.68069

[Cecil and Ryan 1985] T. E. Cecil and P. J. Ryan, Tight and taut immersions of manifolds, Research Notes in Mathematics 107, Pitman (Advanced Publishing Program), Boston, 1985. MR 87b:53089 Zbl 0596.53002

[Dubrovin et al. 1990] B. A. Dubrovin, A. T. Fomenko, and S. P. Novikov, Modern geometrymethods and applications. Part III. Introduction to homology theory, Graduate Texts in Mathematics 124, Springer, New York, 1990. MR 91j:55001 Zbl 0703.55001

[Federer 1969] H. Federer, Geometric measure theory, Die Grundlehren der mathematischen Wissenschaften 153, Springer, New York, 1969. MR 41 \#1976 Zbl 0176.00801

[Hughes and Ranicki 1996] B. Hughes and A. Ranicki, Ends of complexes, Cambridge Tracts in Mathematics 123, Cambridge University Press, Cambridge, 1996. MR 98f:57039 Zbl 0876.57001 
[Kuiper 1984] N. H. Kuiper, "Geometry in total absolute curvature theory", pp. 377-392 in Perspectives in mathematics, edited by W. Jäger et al., Birkhäuser, Basel, 1984. MR 86h:53062 Zbl 0557.53032

[Ozawa 1986] T. Ozawa, "On critical sets of distance functions to a taut submanifold", Math. Ann. 276:1 (1986), 91-96. MR 87k:53142 Zbl 0583.53052

[Pinkall 1984] U. Pinkall, Letter to T. Cecil, 1984.

[Pinkall 1986] U. Pinkall, "Curvature properties of taut submanifolds", Geom. Dedicata 20:1 (1986), 79-83. MR 87d:53111 Zbl 0577.53045

[Ryan 1969] P. J. Ryan, "Homogeneity and some curvature conditions for hypersurfaces", Tôhoku Math. J. (2) 21 (1969), 363-388. MR 40 \#6458 Zbl 0185.49904

[Schoen and Yau 1994] R. Schoen and S.-T. Yau, Lectures on differential geometry, Conference Proceedings and Lecture Notes in Geometry and Topology, I, International Press, Cambridge, MA, 1994. MR 97d:53001 Zbl 0830.53001

[Shiota 1987] M. Shiota, Nash manifolds, Lecture Notes in Mathematics 1269, Springer, Berlin, 1987. MR 89b:58011 Zbl 0629.58002

[Singley 1975] D. H. Singley, "Smoothness theorems for the principal curvatures and principal vectors of a hypersurface", Rocky Mountain J. Math. 5 (1975), 135-144. MR 51 \#4120 Zbl 0303.53018

[Spanier 1966] E. H. Spanier, Algebraic topology, McGraw-Hill Book Co., New York, 1966. MR 35 \#1007 Zbl 0145.43303

[Terng 1985] C.-L. Terng, "Isoparametric submanifolds and their Coxeter groups", J. Differential Geom. 21:1 (1985), 79-107. MR 87e:53095 Zbl 0615.53047

[Thorbergsson 1983] G. Thorbergsson, "Dupin hypersurfaces”, Bull. London Math. Soc. 15 (1983), 493-498. MR 85b:53066 Zbl 0529.53044

Received March 16, 2007. Revised December 10, 2007.

Thomas E. CECIL

Department of MATHEMAtics ANd Computer SCIENCE

College of THE Holy Cross

WORCESTER, MA 01610-2395

UNITED STATES

cecil@mathcs.holycross.edu

QUO-SHIN CHI

DePaRtMent of MATHEMATiCs

CAMPUS BOX 1146

WASHINGTON UNIVERSITY

ST. LOUIS, MO 63130

UNITED STATES

chi@math.wustl.edu

GARY R. JENSEN

DePartment OF MATHEMATICS

CAMPUS BOX 1146

WASHINGTON UNIVERSITY

ST. LOUIS, MO 63130

UNITED STATES 
gary@math.wustl.edu 